\title{
Microstructural Influence on Deformation and Fatigue Life of Composites Using the Generalized Method of Cells
}

\author{
S. M. Arnold ${ }^{1}$, P. Murthy ${ }^{2}$, B.A. Bednarcyk ${ }^{3}$ and E. J. Pineda ${ }^{4}$ \\ NASA Glenn Research Center, Cleveland, $\mathrm{OH}, 44135$
}

\begin{abstract}
A fully coupled deformation and damage approach to modeling the response of composite materials and composite laminates is presented. It is based on the semi-analytical generalized method of cells (GMC) micromechanics model as well as its higher fidelity counterpart, HFGMC, both of which provide closed-form constitutive equations for composite materials as well as the micro scale stress and strain fields in the composite phases. The provided constitutive equations allow GMC and HFGMC to function within a higher scale structural analysis (e.g., finite element analysis or lamination theory) to represent a composite material point, while the availability of the micro fields allow the incorporation of lower scale sub-models to represent local phenomena in the fiber and matrix. Further, GMC's formulation performs averaging when applying certain governing equations such that some degree of microscale field accuracy is surrendered in favor of extreme computational efficiency, rendering the method quite attractive as the centerpiece in a integrated computational material engineering (ICME) structural analysis; whereas HFGMC retains this microscale field accuracy, but at the price of significantly slower computational speed. Herein, the sensitivity of deformation and the fatigue life of graphite/epoxy PMC composites, with both ordered and disordered microstructures, has been investigated using this coupled deformation and damage micromechanics based approach. The local effects of fiber breakage and fatigue damage are included as sub-models that operate on the microscale for the individual composite phases. For analysis of laminates, classical lamination theory is employed as the global or structural scale model, while GMC/HFGMC is embedded to operate on the microscale to simulate the behavior of the composite material within each laminate layer. A key outcome of this study is the statistical influence of microstructure and micromechanics idealization (GMC or HFGMC) on the overall accuracy of unidirectional and laminated composite deformation and fatigue response.
\end{abstract}

\section{Introduction}

Experimental micrographs of composite microstructures have shown that actual microstructures Crarely resemble ordered arrangements and show at least some degree of spatial randomness (see Figure 1 for an example of a polymer matrix composite). However, due to the diminishing effect of microscale randomness at higher length scales, microstructural variability is often ignored and micromechanics based models assuming periodic boundary conditions, with an ordered array of fibers (either square packed or hexagonally packed), are typically utilized. Researchers have investigated the effect of random or disordered microstructures on various composite behaviors, assuming elastic and damage behavior (Trias et al., 2006; Huang et al., 2008; Maligno et al., 2009; Wang et al., 2011; Romanov et al., 2013). Wang et al. (2011) and Trias et al. (2006) focused on the generation of random

\footnotetext{
${ }^{1}$ Technical Lead: Multiscale Modeling, 21000 Brookpark Rd., MS 49-7, Steven.M.Arnold@nasa.gov, AIAA Member

2 Aerospace Research Engineer, 21000 Brookpark Rd., MS 49-7

3

3 Materials Research Engineer, 21000 Brookpark Rd., MS 49-7, AIAA Associate Fellow.

4 Aerospace Research Engineer, 21000 Brookpark Rd., MS 49-7, AIAA Member.
} 
distributions of fibers and quantified their elastic and failure effects using a two dimensional representative volume element (RVE) finite element method (FEM) model loaded in transverse tension. These authors found that as the disorder in the microstructure increased, so did the tensile modulus. This was attributed to higher fiber stresses in the random microstructure when compared to the ordered microstructure. Huang et al. (2008) developed a three dimensional RVE model for the purpose of studying the effects of transverse tensile, shear, and thermal loading on the elastic behavior (e.g., traction, stress concentration, and stress invariant distributions) for ordered and random microstructures of varying volume fractions and loading angles. One conclusion the authors reached is that the range in stress invariant distribution is wider for a disordered (i.e., random fiber array) compared to an ordered array due to irregularity in inter-fiber distance; the effect being lower predicted strength. Maligno et al. (2009) investigated the local elastic and damage evolution effects of inter-fiber spacing in unidirectional fiber-reinforced composites using an RVE comprised of three partial fibers. The authors found that the inter-fiber spacing and residual stress play an important role in damage initiation and evolution. Similarly, Garnich et al. (2013) showed significant differences in fatigue life predictions of a transversely loaded unidirectional PMC depending upon whether one assumes ordered hexagonally packed or disordered microstructures.

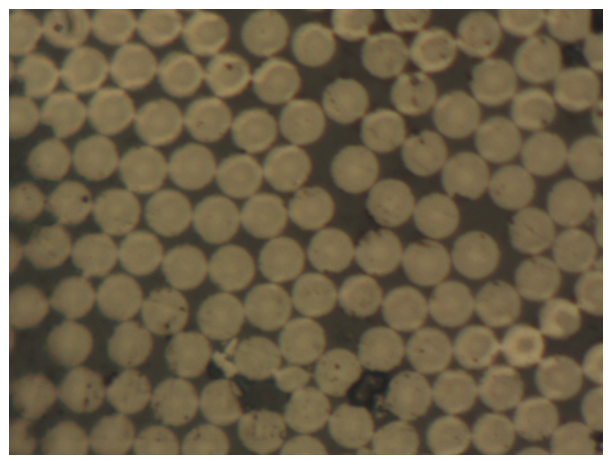

Figure 1: Micrograph of polymer matrix composite

With the increased emphasis on reducing the cost and time to market of new materials, Integrated Computational Materials Engineering (ICME) has become a fast growing discipline within materials science and engineering. ICME is an integrated approach to the design of products and the materials which comprise them by linking material models at multiple time and length scales; such that manufacturing processes, which produce internal material structures that in turn influence material properties and allowables, can be tailored (engineered) to specific industrial applications. In this paper we will utilize the general, synergistic, multiscale-modeling framework for composites, developed by the NASA Glenn Research Center (GRC) and known as MAC/GMC and FEAMAC, see Bednarcyk and Arnold (2002) and Aboudi et al. (2013). This framework can be effectively utilized to link the material microstructure (e.g., constituent phase properties, volume fraction, fiber packing) to ply/laminate properties (mesoscale) and finally to performance (at the macroscale), see Figure 2, in an efficient and accurate manner to enable "fit-for-purpose" tailoring of the composite material. The ability to localize and homogenize between scales with efficiency and accuracy makes MAC/GMC and FEAMAC ideal candidates for ICME simulations in a multiscale environment in which the microstructure can be optimized spatially based on the local loading and environmental history (Pineda et. al. (2014)). Specifically, in this study we will investigate the statistical influence of microstructure (both ordered and disordered) on the overall accuracy of predicted unidirectional and laminated composite effective properties and fatigue life. In addition to examining microstructure-property-performance relationships, prior work (Pineda et al. (2013)) suggests the importance of examining the accuracy of the 
micromechanics idealization (Generalized Method of Cells (GMC) or High Fidelity Generalized Method of Cells (HFGMC)) when considering microstructural arrangement.

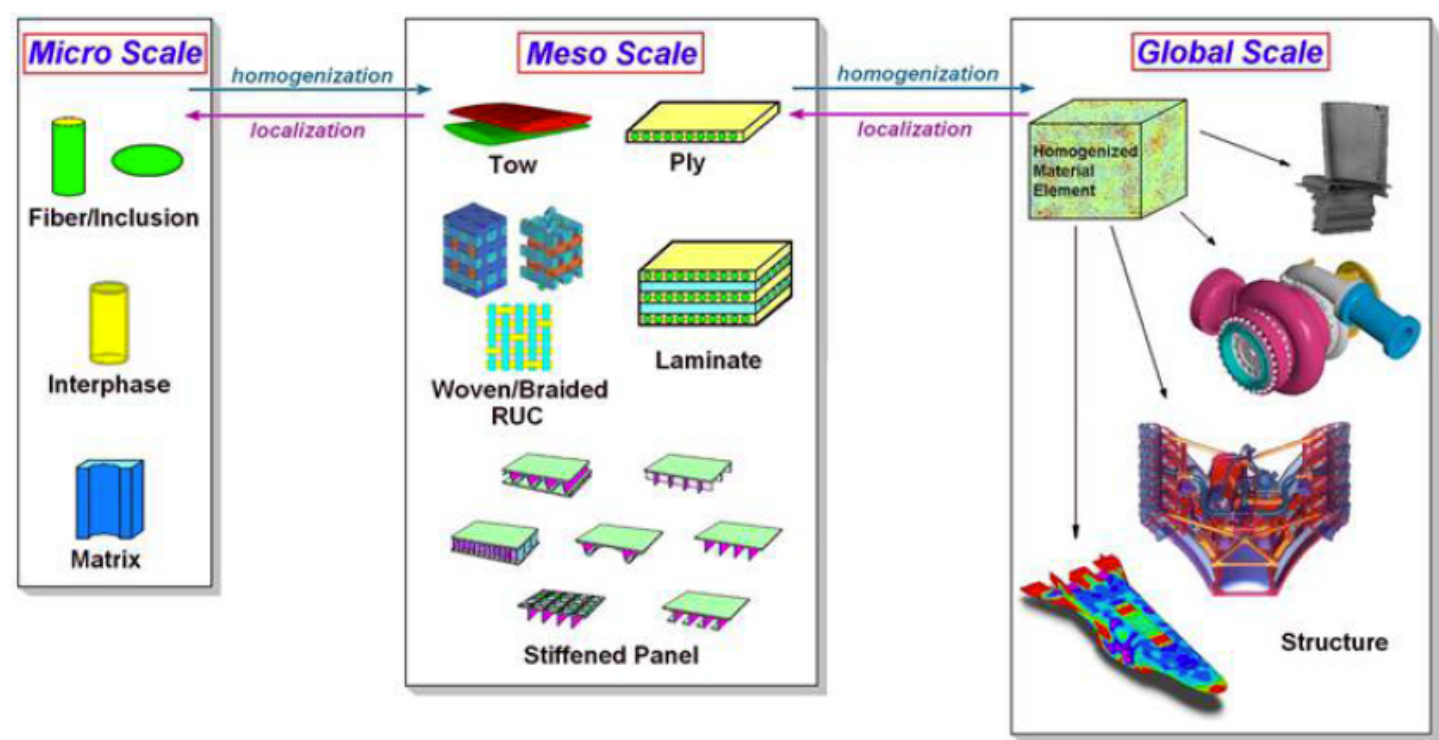

Figure 2: Illustration of relevant levels of scales for multiscale composite analysis.

\section{Generalized Method of Cells}

GMC, first developed by Paley and Aboudi (1992) and HFGMC, first developed by Aboudi et al. (2002), are semi-analytical in nature, and their formulation involves application of several governing conditions in an average sense. It provides the local fields in composite materials, allowing incorporation of arbitrary inelastic constitutive models with various deformation and damage constitutive laws. The microstructure of a periodic material, within the context of GMC and HFGMC, is represented by a rectangular (doubly-periodic) or parallelepiped (triply-periodic) repeating unit cell (RUC) consisting of an arbitrary number of subcells, each of which may be a distinct material (Figure 3 ). In the case of GMC the displacement field is assumed linear, whereas in the case of HFGMC the displacement approximations are assumed quadratic, thus leading to a constant and linear subcell strain field, respectively. In fact it is precisely this higher order assumption in the displacement field that enables HFGMC to retain its ability to compute nonzero transverse shear stress distributions within the composite (i.e., normal and shear coupling), which is so important when dealing with disordered microstructures (Liu and Ghoshal (2014)). However it is also this high-order field assumption, which makes HFGMC more computationally expensive and subject to subcell discretization dependence as compared to GMC.

Displacement and traction continuity is enforced in an average, or integral sense at each of the subcell interfaces and the periodic boundaries of the RUC. These continuity conditions are used to formulate a strain concentration matrix $A$, which gives all the local subcell strains $\left(\boldsymbol{\epsilon}_{s}\right)$ in terms of the global, average, applied strains $\boldsymbol{\epsilon}_{\text {applied }}$ (i.e., $\boldsymbol{\epsilon}_{\boldsymbol{S}}=\mathbf{A} \boldsymbol{\epsilon}_{\text {applied }}$ ). The local subcell stresses $(\boldsymbol{\sigma})$ can then be calculated using the local constitutive law and the local subcell strains. Finally the overall RUC stiffness is obtained utilizing the local constitutive law and the strain concentration matrix averaged over the RUC dimensions. The detailed methodology of GMC and HFGMC and the formulation to be embedded within classical laminate theory is described thoroughly in Aboudi et al. (2013). Also in this reference the 
superior accuracy of HFGMC over that of GMC is demonstrated, consequently in this study HFGMC will be assumed to provide better predictions.

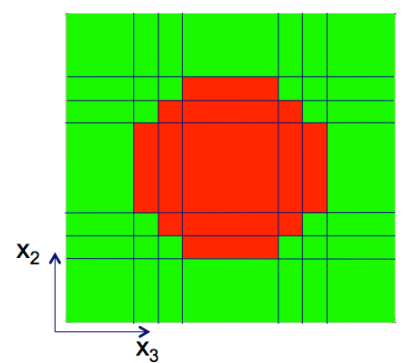

a) Doubly-periodic

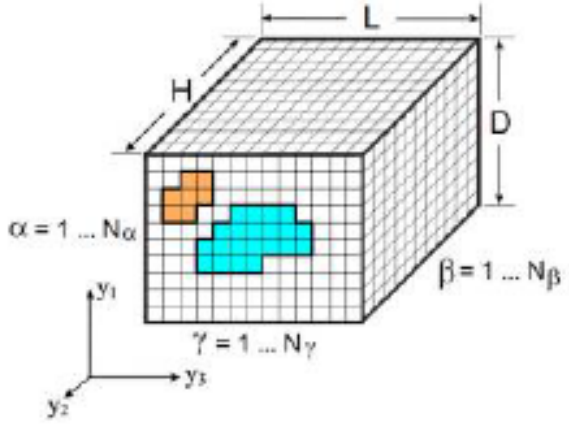

b) Triply Periodic

Figure 3: Composite with repeating microstructure and arbitrary constituents.

\section{Continuum Fatigue Damage Model}

The fatigue life of the composite will be predicted utilizing micromechanics and the isotropic form of the multiaxial, isothermal, continuum damage mechanics model of Arnold and Kruch (1994) for the matrix constituent. When reduced to its isotropic form (the parameters $\omega_{u}, \omega_{f l}, \omega_{m}, \eta_{u}, \eta_{f l}$, and $\eta_{m}$ are set equal to one) this model reduces to the Non-Linear Cumulative Damage Rule (NLCDR) developed at ONERA (Chaboche and Lesne (1988)). This model assumes a single scalar internal damage variable, $D$, that has a value of zero for undamaged material and one for a completely damaged (failed) material. The implementation of the damage model within GMC and HFGMC has been performed on the local scale, thus damage evolves in a given subcell based on the local stress state and number of cycles. For a given damage level, the stiffness of the subcell is degraded by $(1-D)$. Further, the implementation allows the application of a local damage increment, $\Delta D$, and then calculates the number of cycles, $N$, required to achieve this local increment of damage. This approach allows the model to determine the stress state in the composite, identify the subcell that will reach the desired damage level in the fewest cycles, apply that number of cycles, and calculate the damage that arises throughout the remainder of the composite. Then the composite can be reanalyzed and a new stress state determined based on the new, spatially varying, damage level throughout the composite RUC. In this way, the local and global stress and damage analyses are coupled. As the damage in the composite evolves, the stress field in the composite is redistributed, which then affects the evolution of damage.

For an isotropic material, the damage parameters that must be selected reduce to $M, \beta$ and $\hat{a}$, and the pertinent equation relating the fatigue life of the isotropic material to the cyclic stress state is,

$$
N_{F}=\frac{\left(\sigma_{u}-\sigma_{\max }\right)\left(\frac{M}{\sigma_{\max }-\bar{\sigma}}\right)^{\beta}}{\hat{a}(1+\beta)\left(\sigma_{\max }-\bar{\sigma}-\sigma_{f l}\right)} \quad \text { for } N_{F}>0
$$

where $\sigma_{u}$ is the material ultimate strength, $\sigma_{f l}$ is the material fatigue limit (stress below which damage does not occur), $\sigma_{\max }$ is the maximum stress during a loading cycle, $\bar{\sigma}$ is the mean stress during a loading cycle, and $N_{F}$ is the number of cycles to failure. Note that, in the terminology of Arnold and 
Kruch (1994), $\hat{a}=a \frac{\sigma_{u}}{\sigma_{f l}}$. Utilizing the above equation, the damage model parameters $M, \beta$ and $\hat{a}$ can be selected for an isotropic material based on the material's S-N curve (stress level vs. cycles to failure). Both the fatigue limit and the scaling parameter $M$ are general enough to account for the effect of mean stress. However in this study this additional effect is ignored since only one $\mathrm{R}$ ratio is examined. An S-N curve for epoxy was obtained from Plastics Design Library (1995), and the fatigue damage model parameters were selected as $M=150 \mathrm{MPa}, \beta=9$, and $\hat{a}=0.05$, with $\sigma_{u}=80 \mathrm{MPa}$, and $\sigma_{f l}=27$ $\mathrm{MPa}$. A plot showing the fatigue model characterization is given in Figure 4.

A second damage model within GMC and HFGMC is much simpler and involves degradation of a material's strength due to cyclic loading. As shown by Wilt et al. (1997), this type of damage model can be used to simulate the fatigue behavior of fibers that occurs in-situ during fatigue of a composite. The model assumes a logarithmic relation between the material's strength and the number of cycles within a certain range such that:

$$
\begin{array}{cc}
\sigma_{u}=\sigma_{u 1} & 0 \leq N \leq N_{1} \\
\sigma_{u}=\sigma_{u 1}-\frac{\left(\sigma_{u 1}-\sigma_{u 2}\right) \log \left(N / N_{1}\right)}{\log \left(N_{2} / N_{1}\right)} & N_{1} \leq N \leq N_{2} \\
\sigma_{u}=\sigma_{u 2} & N_{2} \leq N
\end{array}
$$

This strength degradation model was employed in the present example to model the longitudinal fatigue behavior of the graphite fiber. The necessary parameters for the model are $\sigma_{u 1}, \sigma_{u 2}, N_{1}$, and $N_{2}$. The values of these parameters chosen for the graphite fiber are shown in Fig. 5 . Note that these data were not correlated with experiment, but rather chosen based on the expected trend. Given these required parameters for the fatigue damage models for each phase in the graphite/epoxy composite, macroscopic or composite fatigue life of both unidirectional and [0/90] cross-ply composite laminates can be simulated. The static deformation response of both fiber and matrix were assumed to be linear elastic, with all damage coming from cyclic fatigue. The associated material properties are given in Table 1. 


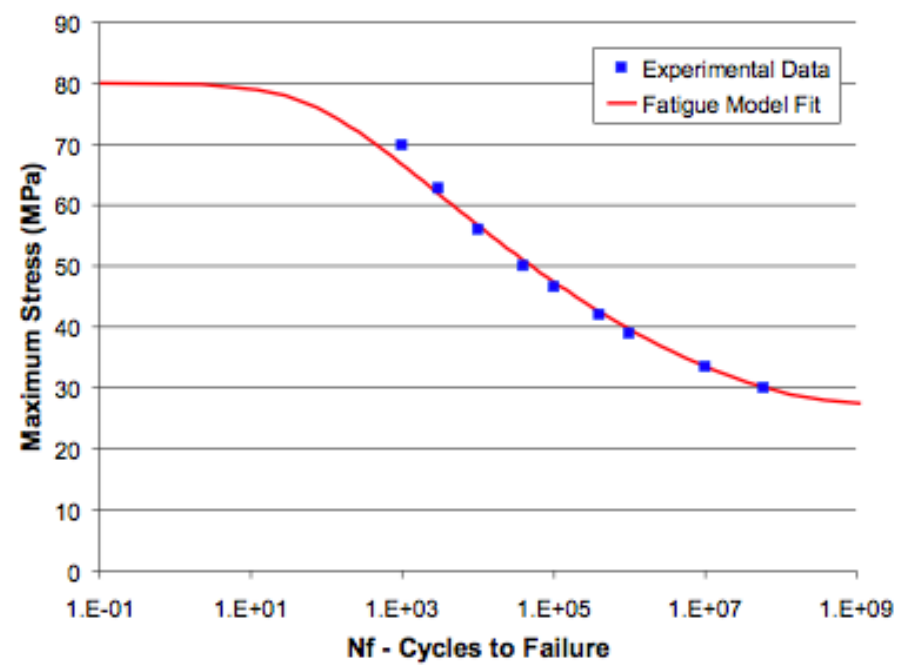

Figure 4: Characterization of the stiffness reduction fatigue damage model parameters for the epoxy matrix. Experimental data are from Plastics Design Library (1995).

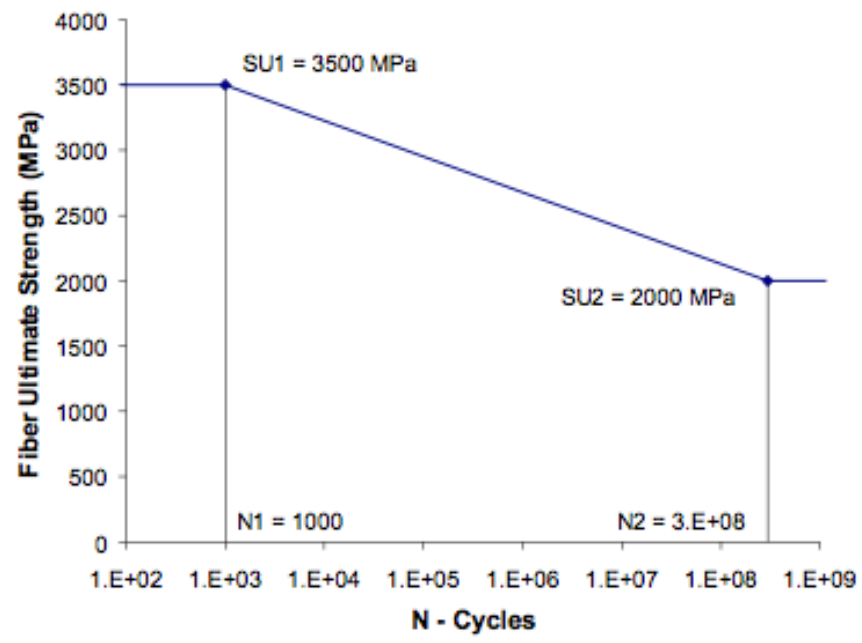

Figure 5: Strength reduction fatigue model parameters assumed for the graphite fiber.

Table 1: Constituent (fiber/matrix) elastic properties

\begin{tabular}{|c|c|}
\hline Graphite Fiber & Epoxy Matrix \\
\hline $\mathrm{E}_{\mathrm{f} 11}=388.2 \mathrm{GPa}$ & $\mathrm{E}_{\mathrm{m}}=3.45 \mathrm{GPa}$ \\
\hline $\mathrm{E}_{\mathrm{f} 22}=7.6 \mathrm{GPa}$ & $v_{\mathrm{m}}=0.35$ \\
\hline$v_{\mathrm{f} 12}=0.41$ & $G_{\mathrm{m} 23}=1.278 \mathrm{GPa}$ \\
\hline$v_{\mathrm{f} 23}=0.45$ & \\
\hline$G_{\mathrm{f} 12}=14.9 \mathrm{GPa}$ & \\
\hline
\end{tabular}




\section{Results}

\section{GMC and HFGMC RUC Refinement: Ordered Microstructure}

The elastic properties of graphite fiber and epoxy matrix are given in Table 1, while the fatigue parameters are given in Section 3. The matrix (represented by green subcells in Figure 6) was assumed isotropic with a Young's modulus $E_{m}$ and a Poisson's ratio $v_{m}$, and the fiber (shown in Figure 6 with red subcells) was assumed transversely isotropic; where $\mathrm{E}_{\mathrm{f} 11}$ is the longitudinal fiber modulus, $\mathrm{E}_{\mathrm{f22}}$ is the transverse fiber modulus, $v_{f 12}$ and $v_{f 12}$ are the longitudinal and transverse fiber Poisson Ratios, and $G_{f 12}$ is the longitudinal (axial) fiber shear modulus in Table 1. Note the ratio of constituent properties are $\mathrm{E}_{\mathrm{fl}} / \mathrm{E}_{\mathrm{m}}$ $=112 ; \mathrm{E}_{\mathrm{ff}} / \mathrm{E}_{\mathrm{m}}=2.2 ; \mathrm{G}_{\mathrm{fL}} / \mathrm{G}_{\mathrm{m}}=11.6 ; \mathrm{G}_{\mathrm{fT}} / \mathrm{G}_{\mathrm{m}}=2.62$. Although it is well known that $\mathrm{GMC}$ (Aboudi et. al. 2013) has no subcell discretization dependence, for a fixed RUC, the RUC can still be refined such that the volume fraction of fiber and matrix can be better represented. The influence of such discretization dependence is shown in Figures 6 and 7, wherein one sees that the transverse normal and transverse and longitudinal shear moduli (effective stiffness's) are only slightly impacted for both GMC and HFGMC (with HFGMC having the larger dependence). The transverse fatigue life (Figure 7), on the other hand, is significantly affected as it depends more heavily on the accuracy of the local fields. Figure 7 indicates that fatigue life is much more sensitive to RUC discretization than effective properties in that the maximum difference in life (from one RUC discretization to another) for GMC is approximately a factor of 2.5, whereas, for HFGMC, the factor is 28 . The difference in life between GMC and HFGMC for a given RUC idealization was typically less than a factor of two, with Arch ID 1 and 13 being exceptions at $5 x$ and $3 x$, respectively. GMC typically predicted longer lives than did HFGMC for a corresponding RUC idealization.

The numerical error between the effective composite properties predicted by HFGMC and GMC are given in Table 2, with the maximum normal stiffness error being less than $2 \%$ while the maximum error in shear stiffness is approximately 30\%. Clearly, the largest difference occurred when using Arch ID 1 (2x2) discretization. This is not surprising since in the case of HFGMC (which has discretization dependence, due to the capturing of local normal and shear coupling) the circular nature of the fiber is not well represented; see Aboudi et al. 2013. Furthermore, as the circular nature of the fiber is better approximated, local stress concentrations within the RUC are better captured, and the fatigue life decreases, with HFGMC being more dramatically impacted than GMC. Note that in all RUC idealizations, except the last one (i.e., $12 \times 12$ ), only two matrix subcell are present between fibers. Comparing the $5 \mathrm{x}$ 5 and $12 \times 12$ RUCs one can see that a further discretization of the matrix between fibers further impacts the life predictions produced by HFGMC as the stress field variation between fibers is captured more accurately. In the subsequent microstructure study, the $12 \times 12 R U C$ will be used as the baseline $R U C$ representing ordered and disordered periodic microstructures. This is primarily done for computational efficiency purposes since, although HFGMC is more accurate, on average it is orders of magnitudes slower than GMC. Therefore the size of the RUCs examined, particularly in fatigue, must be limited. 


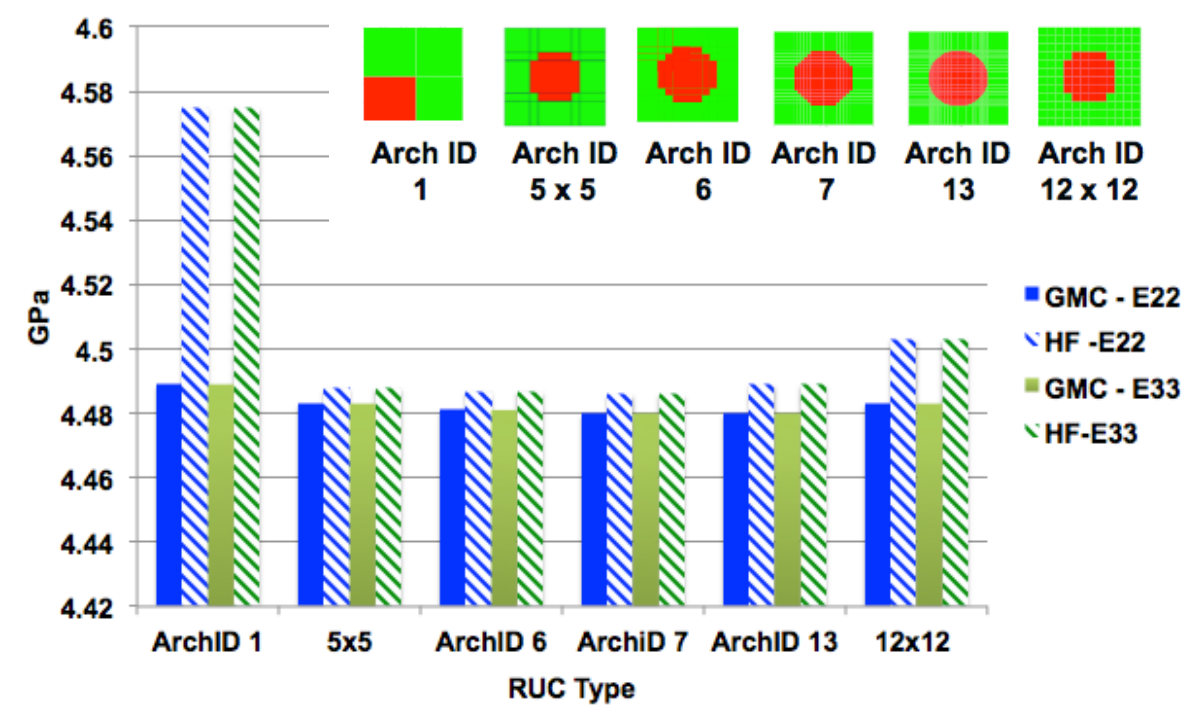

a) Transverse Moduli (Note scale has been greatly enhanced)

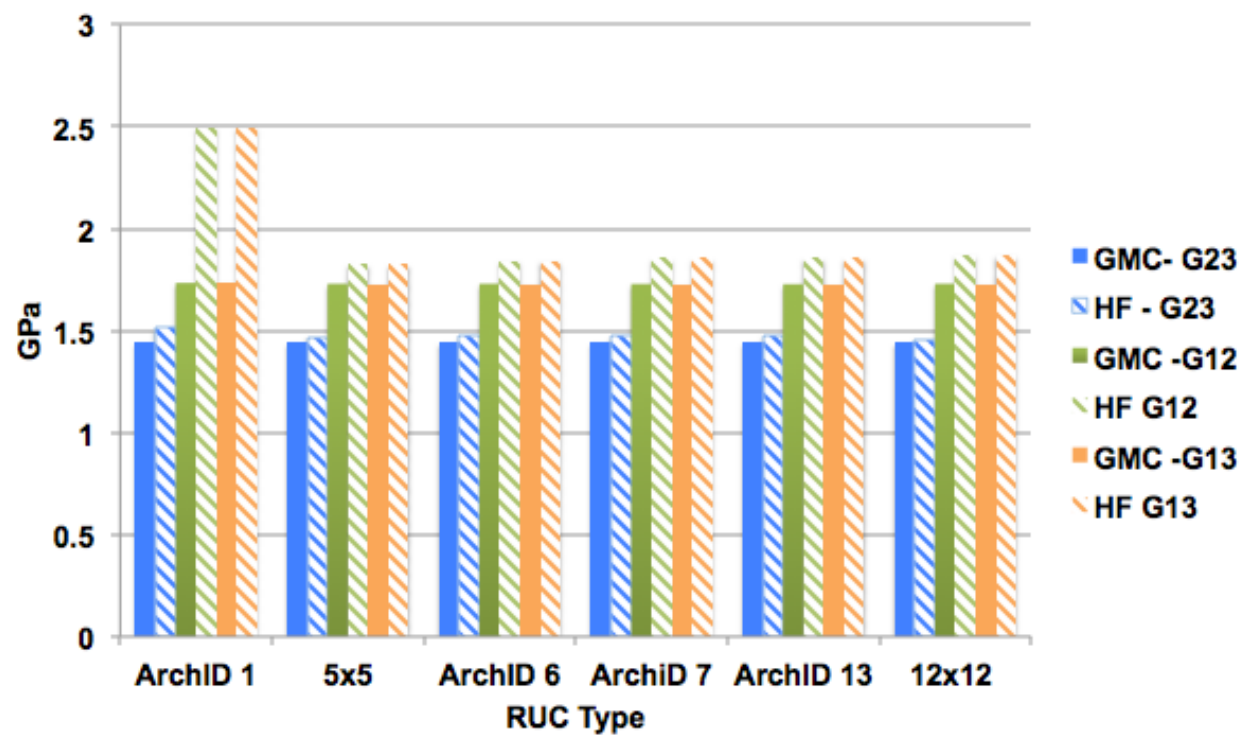

b) Transverse (G23) and Longitudinal (G12, G13) Shear Modulus

Figure 6: Effective Property prediction for multiple ordered RUC discretizations, given a $22 \%$ fiber volume fraction. 


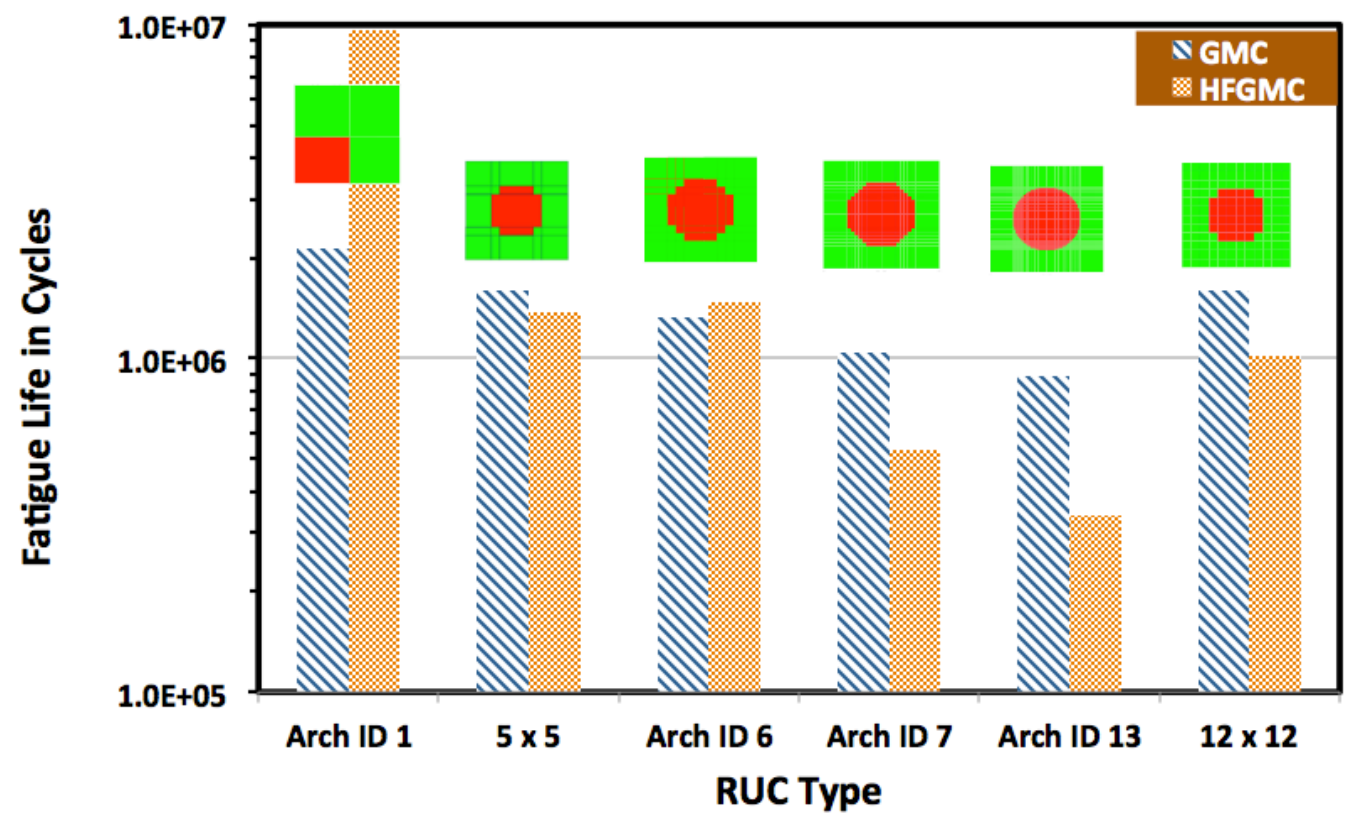

Figure 7: Predicted fatigue life for a unidirectional, transversely loaded, PMC composite (22\% fiber volume fraction, $\sigma_{\max }=40 \mathrm{MPa}, \mathrm{R}=0.1$ ).

Table 2 Difference between HFGMC and GMC effective property predictions as a function of RUC discretization.

\begin{tabular}{|c|c|c|c|c|c|c|c|c|c|}
\hline RUC Type & E11 & E22 & E33 & v23 & v12 & v13 & G23 & G12 & G13 \\
\hline ArchID 1 & 0.0001 & 0.0188 & 0.0188 & -0.012 & 0.0027 & 0.0027 & 0.0469 & 0.3061 & 0.3061 \\
\hline 5x5 & 0 & 0.0011 & 0.0011 & 0.0004 & 0.0008 & 0.0008 & 0.019 & 0.0526 & 0.0526 \\
\hline ArchID 6 & 0 & 0.0013 & 0.0013 & 0.0004 & 0.0008 & 0.0008 & 0.0197 & 0.0608 & 0.0608 \\
\hline ArchiD 7 & 0 & 0.0013 & 0.0013 & 0.0012 & 0.0011 & 0.0011 & 0.0217 & 0.0684 & 0.0684 \\
\hline ArchID 13 & 0 & 0.002 & 0.002 & 0.0004 & 0.0011 & 0.0011 & 0.0217 & 0.069 & 0.069 \\
\hline 12x12 & 0 & 0.0044 & 0.0044 & -0.002 & 0.0011 & 0.0011 & 0.0123 & 0.0759 & 0.0759 \\
\hline
\end{tabular}

\section{Ordered versus Disordered Microstructures}

Given a fixed, single fiber $12 \times 12$ RUC (ordered, square packed microstructure), as well as RUCs with multiple fibers with the same level of discretization, the influence of random fiber placement (disordered microstructure) can be examined. As the number of fibers within a given RUC increase, this RUC can approach what is termed a statistical RUC. Figure 8 illustrates three cases for a 4 fiber RUC; Case 1: ordered (this case gives identical answers to a single fiber RUC with periodic boundary conditions), while Cases 2 and 3 are disordered. In Figure 8 we have shown the actual RUCs which have periodic boundary conditions applied to them and the tiled (expanded) version of Case 2 to better illustrate the actual microstructure being simulated for that case. Table 3 provides results (effective composite properties and number of cycles to end of life) from both GMC and HFGMC micromechanics analyses. Although unrealistic for PMCs, a volume fraction of $22 \%$ was assumed, since as volume fraction increases the ability to move fibers decreases. Clearly, the longitudinal stiffnesses, $E_{11}$, are identical for both GMC and HFGMC, while all other normal moduli and Poisson's ratios are very close (less than 1.5\% difference). The largest variations occur in the longitudinal shear moduli (G12 and G13), approximately $7-15 \%$ depending upon the case, whereas the transverse shear moduli (G23) differ by no 
more than $3 \%$ between GMC and HFGMC. Note, Case 2 produces the highest discrepancy between HFGMC and GMC. Also, as will be shown in a future publication, the actual percentage discrepancy is highly dependent upon volume fraction and property mismatch between constituents. However, the trends indicated herein remain the same.

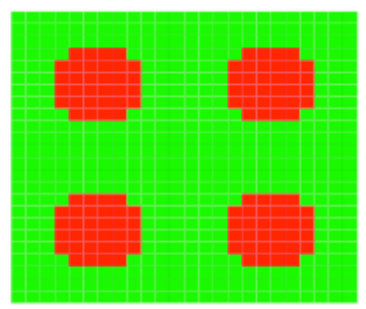

Case 1

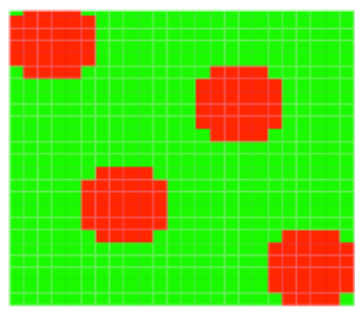

Case 3

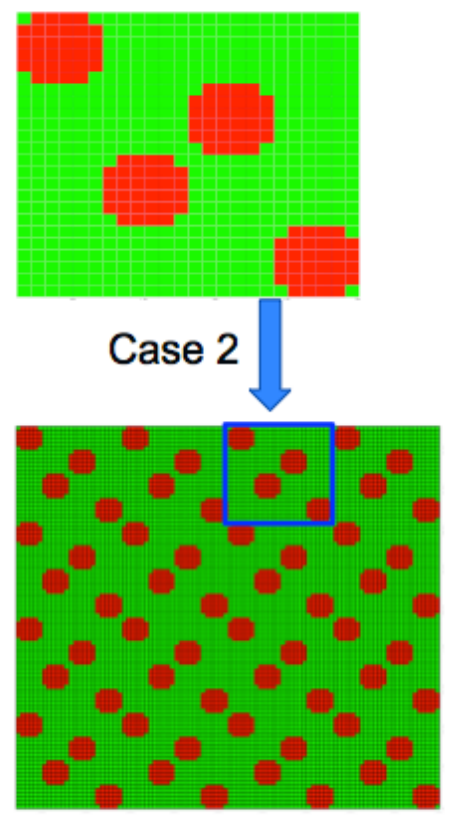

Case 2: Tiled

Figure 8: Square-packed $24 \times 24$ RUC subcell architecture. Red subcells=Fiber, green subcells= matrix

Before considering failure, it would be informative to assess the influence of the number of fibers within a given RUC (e.g., 4 fibers: 24 × 24; 9 fibers: $36 \times 36$; 25 fibers: 60 x 60, and 100 fibers: $120 \times 120$ subcells, see Fig. 9) on the predicted effective properties, when utilizing either the GMC or HFGMC idealization. Table 4 and 5 provide these results. Table 4 illustrates the impact of considering additional fibers for the case of random perturbations of a square pack microstructure (defined as slightly random) whereas Table 5 assesses the case of truly random microstructures. Clearly, as more fibers are added (tending toward a statistical RUC), all properties tend to converge. One measure of convergence (for the case of truly random, Table 5) is whether or not transverse isotropy can be recovered such that E22 $=\mathrm{E} 33, \mathrm{G} 12=\mathrm{G} 13$, and $\mathrm{G} 23=\mathrm{E} 22 /(2(1+v 23)$. Although transverse isotropy is not expected to be recovered in the case of slight perturbations of square pack (see Table 4), the properties appear to converge for an RUC containing 9 or more fibers. Similarly, it is apparent that predicted in-plane normal moduli (E22 and E33) and longitudinal shear moduli are slightly different $(<1 \%$ and approximately $2.5 \%$, respectively when using GMC; and less than $0.2 \%$ for all when using HFGMC) when slight perturbations are present as compared to those obtained from an ordered periodic microstructure. Consequently, in practicality 4 fiber RUCs should be sufficient to investigate the influence of disorder on the two micromechanics approaches. In the case of truly random microstructures, the computed G23 modulus matches ( $<0.5 \%$ error) that obtained from the isotropy assumption when 25 fibers are used in the case of GMC and 9 fibers in the case of HFGMC. Four fibers produce in-plane modulus agreement within $0.8 \%$ when using HFGMC. Another potential measure is to create probability density functions (PDFs) for each property and determine the number of fibers required to minimize the variation about the 
mean value. Although not shown here, this was done and it was determined that 100 or more fibers would be required to retrieve "deterministic" effective properties.

Table 3: Elastic effective composite properties and transverse cycles to failure

\begin{tabular}{|c|c|c|c|c|c|c|}
\hline \multicolumn{3}{|c|}{4 Fiber: 24x24 Base cell Models; Vf=22\% } & \multirow{2}{*}{\multicolumn{2}{|c|}{ Case 2}} & \multirow{2}{*}{\multicolumn{2}{|c|}{ Case 3}} \\
\hline & & & & & & \\
\hline Property & GMC & HFGMC & GMC & HFGMC & GMC & HFGMC \\
\hline E11S & 88950 & 88950 & 88950 & 88950 & 88950 & 88950 \\
\hline N12S & 0.3639 & 0.3643 & 0.363 & 0.364 & 0.363 & 0.364 \\
\hline N13S & 0.364 & 0.3643 & 0.363 & 0.364 & 0.363 & 0.364 \\
\hline E22S & 4483 & 4503 & 4380 & 4443 & 4388 & 4454 \\
\hline N23S & 0.508 & 0.5073 & 0.518 & 0.514 & 0.517 & 0.513 \\
\hline E33S & 4483 & 4503 & 4380 & 4443 & 4388 & 4454 \\
\hline G23S & 1442 & 1460 & 1442 & 1487 & 1442 & 1481 \\
\hline G13S & 1730 & 1872 & 1607 & 1893 & 1615 & 1886 \\
\hline G12S & 1730 & 1872 & 1607 & 1893 & 1615 & 1886 \\
\hline Life & $1,590,152$ & $1,018,736$ & $3,605,070$ & $2,930,013$ & $2,428,430$ & $2,903,263$ \\
\hline
\end{tabular}
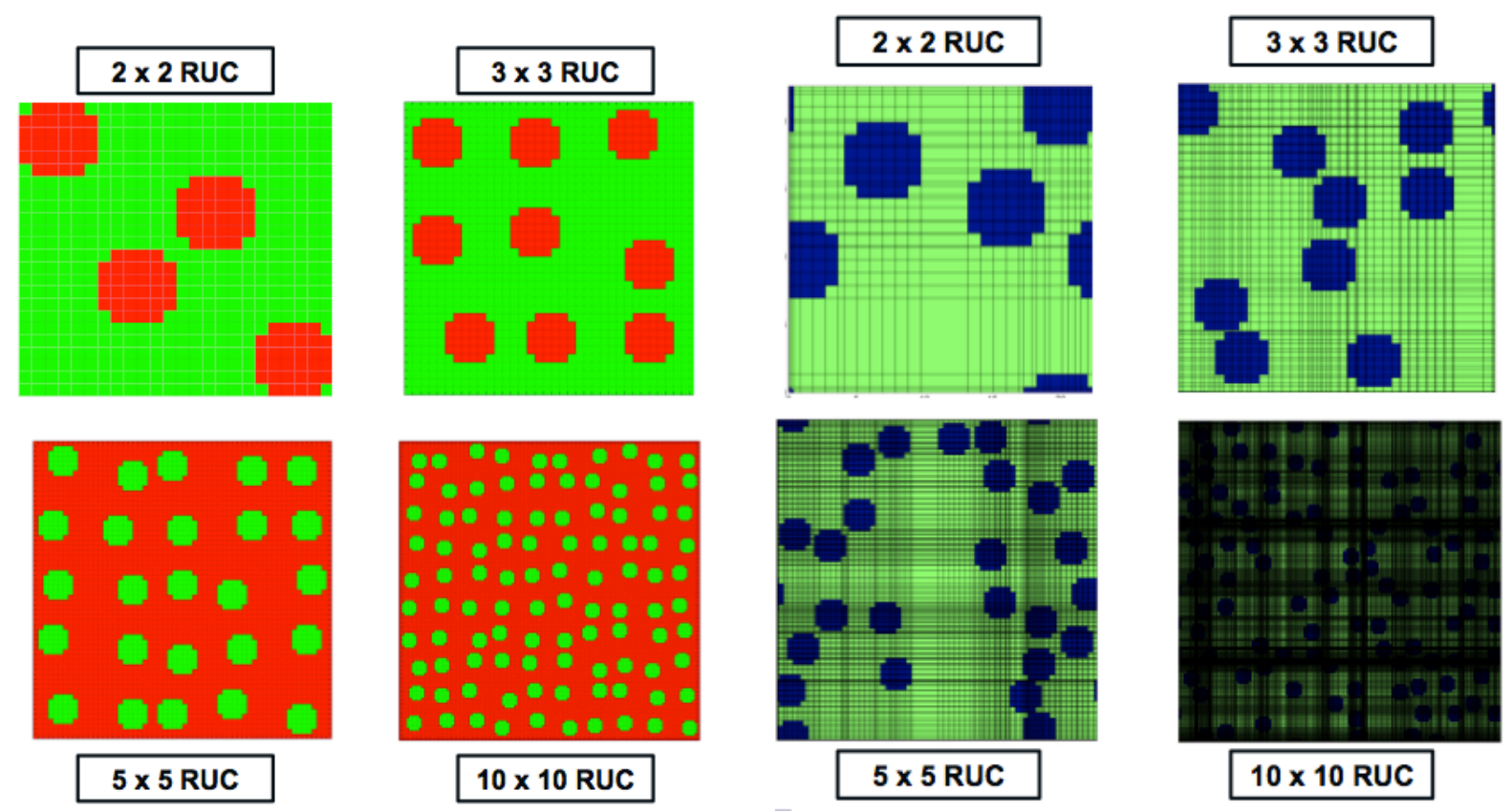

$5 \times 5$ RUC

a) Slight Perturbation on Square Pack

b) Truly random architecture

Figure 9 Illustrations of Multi-fiber Representative Unit Cells (RUCs) 
Table 4 Mean property values of all runs with 4, 9, 25, and 100 fibers within RUC: Slightly perturbed

\begin{tabular}{|c|c|c|c|c|c|c|c|c|c|c|}
\hline \# Fibers & E11 & v12 & v13 & E22 & v23 & E33 & G23 & G13 & G12 & \multirow{6}{*}{ GMC } \\
\hline 1 & 88950 & 0.3639 & 0.3639 & 4483.0 & 0.5082 & 4483.0 & 1442.0 & 1730.0 & 1730.0 & \\
\hline 4 & 88950 & 0.3638 & 0.3638 & 4454.2 & \begin{tabular}{|l|}
0.5107 \\
\end{tabular} & \begin{tabular}{|l|}
4455.2 \\
\end{tabular} & 1442.0 & \begin{tabular}{|l|}
1699.2 \\
\end{tabular} & 1696.2 & \\
\hline 9 & 88950 & 0.3637 & 0.3638 & 4445.8 & \begin{tabular}{|l|}
0.5114 \\
\end{tabular} & \begin{tabular}{|l|}
4447.6 \\
\end{tabular} & 1442.0 & \begin{tabular}{|l|}
1690.8 \\
\end{tabular} & 1685.1 & \\
\hline 25 & 88950 & 0.3637 & 0.3637 & 4438.5 & \begin{tabular}{|l|}
0.5123 \\
\end{tabular} & \begin{tabular}{|l|}
4438.7 \\
\end{tabular} & 1442.0 & \begin{tabular}{|l|}
1678.3 \\
\end{tabular} & 1677.9 & \\
\hline 100 & 88950 & 0.3637 & 0.3637 & 4433.2 & \begin{tabular}{|l|}
0.5128 \\
\end{tabular} & \begin{tabular}{|l|}
4433.2 \\
\end{tabular} & 1442.0 & \begin{tabular}{|l|}
1671.5 \\
\end{tabular} & 1671.5 & \\
\hline \multicolumn{11}{|r|}{$V f=22 \%$} \\
\hline 1 & 88950 & 0.3643 & 0.3643 & 4503.0 & \begin{tabular}{|l|}
0.5073 \\
\end{tabular} & 4503.0 & 1460.0 & \begin{tabular}{|l|}
1872.0 \\
\end{tabular} & 1872.0 & \multirow{5}{*}{ HFGMC } \\
\hline 4 & 88950 & 0.3643 & 0.3643 & 4493.9 & \begin{tabular}{|l|}
0.5083 \\
\end{tabular} & 4493.7 & 1463.3 & \begin{tabular}{|l|}
1874.1 \\
\end{tabular} & 1875.4 & \\
\hline 9 & 88950 & 0.3643 & 0.3643 & 4494.1 & \begin{tabular}{|l|}
0.5081 \\
\end{tabular} & 4494.8 & 1462.9 & 1877.1 & 1873.1 & \\
\hline 25 & 88950 & 0.3643 & 0.3643 & 4493.9 & \begin{tabular}{|l|}
0.5082 \\
\end{tabular} & 4493.9 & 1463.0 & 1875.5 & 1875.3 & \\
\hline 100 & 88950 & 0.3643 & 0.3643 & 4493.7 & \begin{tabular}{|l|}
0.5083 \\
\end{tabular} & 4493.7 & 1463.1 & \begin{tabular}{|l|}
1875.5 \\
\end{tabular} & 1875.3 & \\
\hline
\end{tabular}

Table 5 Mean property values of all runs with 4, 9 and 25 fibers within RUC: Truly random

\begin{tabular}{|c|c|c|c|c|c|c|c|c|c|c|}
\hline \# Fibers & E11 & v12 & v13 & E22 & v23 & E33 & G23 & G13 & G12 & \multirow{4}{*}{ GMC } \\
\hline 4 & 88881 & 0.3637 & 0.3636 & 4429.1 & 0.5132 & 4428.1 & 1441.9 & 1669.6 & 1671.8 & \\
\hline 9 & 88893 & 0.3635 & 0.3636 & 4410.0 & \begin{tabular}{|l|}
0.5147 \\
\end{tabular} & 4411.1 & 1441.9 & 1647.8 & 1643.8 & \\
\hline 25 & 88912 & 0.3634 & 0.3635 & 4397.6 & 0.5159 & 4398.2 & 1442.0 & 1630.2 & 1628.4 & \\
\hline & & & & & & & & & & $V f=22 \%$ \\
\hline 4 & 88882 & 0.3643 & 0.3643 & 4477.5 & 0.5100 & 4478.1 & 1471.3 & 1903.9 & 1901.1 & \multirow{3}{*}{ HFGMC } \\
\hline 9 & 88895 & 0.3643 & 0.3644 & 4475.3 & 0.5103 & 4476.1 & 1473.6 & 1917.6 & 1910.9 & \\
\hline 25 & 88914 & 0.3643 & 0.3644 & 4476.8 & 0.5102 & 4477.9 & 1474.6 & 1927.5 & 1919.8 & \\
\hline
\end{tabular}

As one might suspect, failure (in this case the transverse fatigue life, see Table 3 ) is greatly influenced by microstructure [126\% (case 2 vs. case 1 ) and $53 \%$ (case 3 vs. case 1 ) for GMC and $188 \%$ and $185 \%$ for HFGMC, respectively] and micromechanics idealization [(56\% (GMC case1 vs. HFGMC case 1 ) and $23 \%$ (GMC case 2 vs. HFGMC case2) and $16 \%$ (GMC case 3 vs. HFGMC case3)] since failure is driven by the local fields (here, because of the damage model being used, these are local, microscale stress fields) ${ }^{5}$.

Classic S-N (applied stress vs. number of cycle to failure) curves are shown in Figure 10 and 11 for unidirectional laminates loaded longitudinally [0] and transversely [90], as well as cross-ply [0/90]s laminates. Figure 11, merely illustrates a zoomed in view of the $[0 / 90]_{s}$ laminate case (two curves labeled as "12×12" with additional points associated with disordered cases). Both GMC and HFGMC simulations are given for ordered microstructures with a fiber volume fraction of $22 \%$. As expected, fatigue lives for [0] laminates are identical regardless of which micromechanics idealization is used, i.e., GMC or HFGMC. However, in the case of [90] or [0/90] laminates, differences between GMC and HFGMC are more pronounced; roughly a factor of 1.5 to 2 for both [90] and [0/90] ${ }_{s}$ depending upon the applied load level. This difference is well within the typical experimental scatter in fatigue. In Figure 11,

\footnotetext{
${ }^{5}$ Note since the largest difference between GMC and HFGMC idealizations occurred for the ordered case - to determine the precise influence of microstructure disorder, in-situ properties in GMC should be adjusted so that both GMC and HFGMC give the same results for the ordered case. This is reserved for a future study.
} 
one can see an averaged difference (approximately 60\%) for all stress levels between GMC and HFGMC predicted lives given an ordered microstructure, case 1; with HFGMC predicting shorter lives.

Now, considering the case of disordered microstructures a few sample cases have been included in Figures 10 and 11; they are indicated by various lone symbols for both the [90] and [0/90] laminate cases. Clearly, lives of the disordered composites are shorter than their ordered counterpart. GMC predicts significantly shorter life than the corresponding GMC ordered case (see larger solid green circle (Fig. 10) for the [90] laminate and the green $X$ and orange diamond (Fig. 11) symbols in the case of $[0 / 90]_{s}$ laminates). HFGMC also produces shorter lives, but to a lesser extent than the corresponding ordered case (see purple solid circle (Fig. 10) and blue square and orange triangle (Fig. 11) symbol). Note that in Fig. 11 two random disordered cases are examined for both GMC and HFGMC; in the case of GMC they give the same lives but in the case of HFGMC they vary by roughly $72 \%$. Consequently, the sensitivity to disorder appears to be greater with HFGMC than it does with GMC. Since HFGMC has been shown to have similar accuracy to FEA under static loading conditions (see Aboudi et al. (2013), Pineda et al (2013)), this brings into question the ability to use GMC to predict fatigue lives for random microstructures. This will be further addressed subsequently.

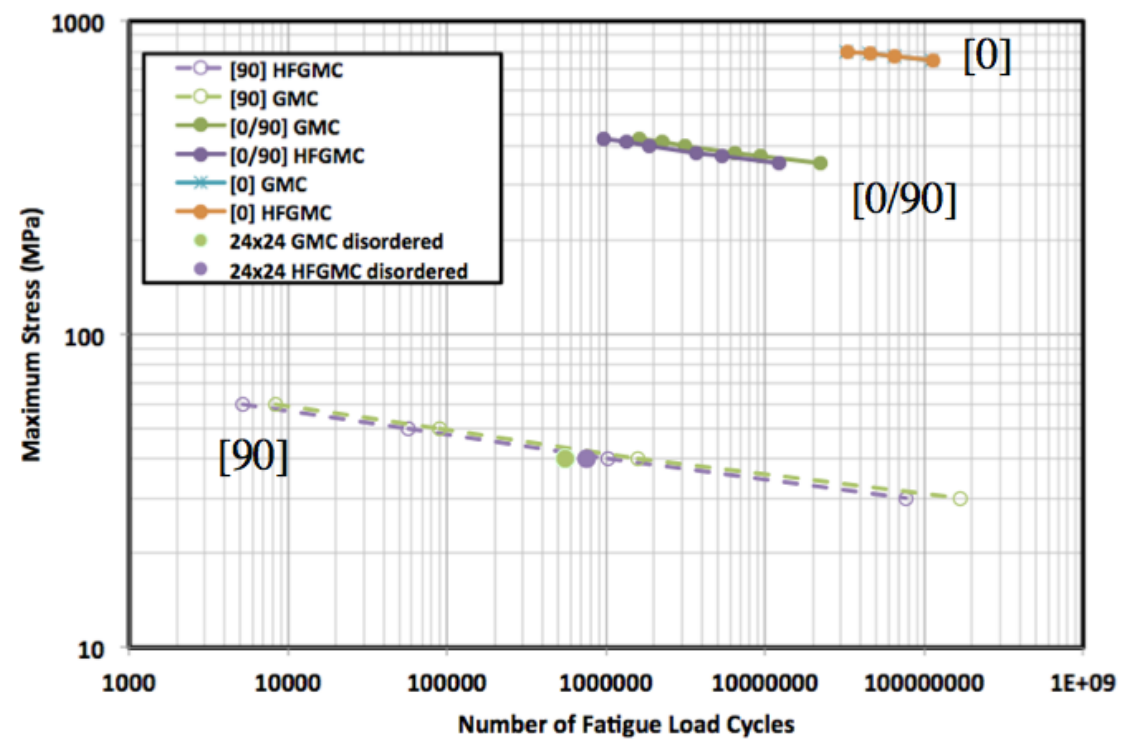

Figure 10: S-N curve for unidirectional [0] and [90] and Cross-ply laminated [0/90] graphite/epoxy composite system, volume fraction $\left(V_{f}\right)=22 \%$. 


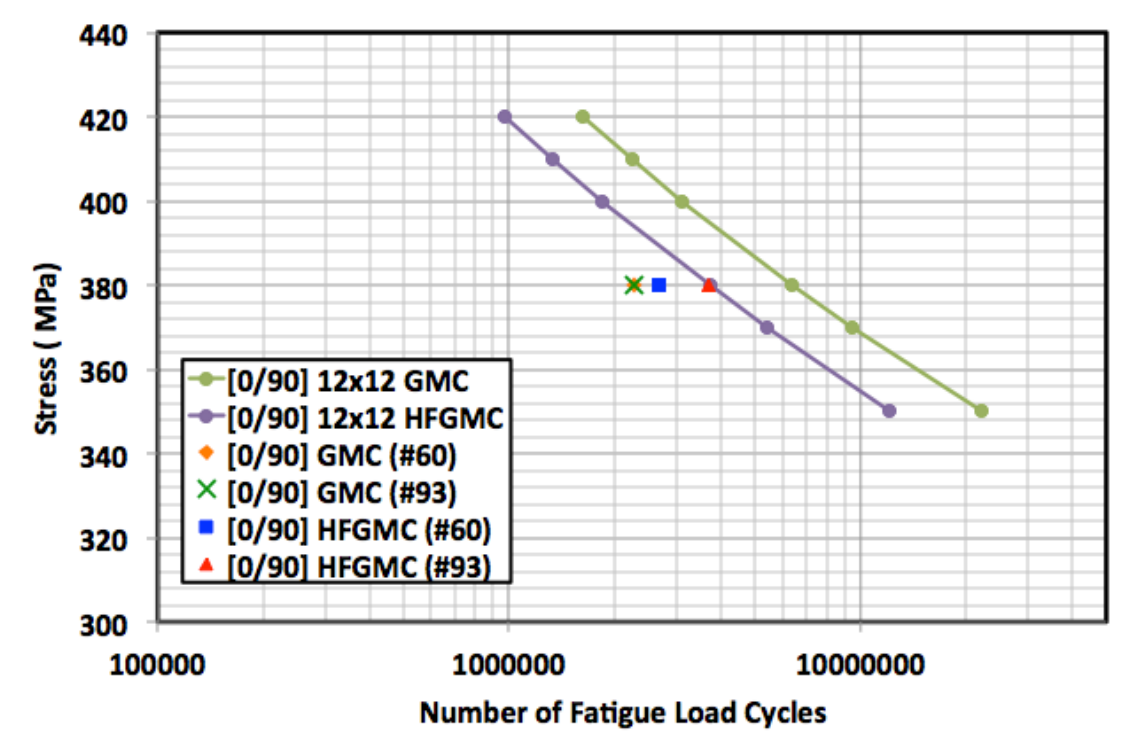

Figure 11: S-N curve for Cross-ply laminated [0/90] graphite/epoxy composite system, $V_{f}=22 \%$.

The additional sensitivity to disordered microstructures in the case of HFGMC as compared to GMC can be attributed to the fact that disordered microstructures induce more local normal/shear coupling and thus cause a higher fluctuation in local fields when compared to ordered microstructures (wherein the spacing between fibers is uniform). Obviously, since the matrix fatigue damage is driven by the local stress state and the local stress field (and therefore damage evolution) is highly dependent upon the given disordered microstructure, shorter lives are clearly possible. This is consistent with previous FEA investigations involving disorder and tensile strength calculations; see Wang et al. (2011) and Trias et al. (2006). GMC is unable to capture these fluctuations in the local stress fields accurately due to the known lack of normal and shear coupling. This lack of normal/shear coupling is magnified when fibers are clustered, i.e., pockets of high volume fractions occur within an RUC. To investigate this potential variation, 100 random perturbations (of a square packed microstructure) were constructed assuming a 4 fiber RUC ( $24 \times 24$ subcell). Note, an aspect ratio of one (between the horizontal and vertical subcell dimensions) was always maintained in order to minimize any discretization dependence in the results. The resulting life as a function of Monte Carlo run number is plotted in Figure 12, where a given run number is associated with the same microstructure analyzed by both GMC and HFGMC. A corresponding probability density function (PDF) is shown in Figure 13 for results coming from GMC and HFGMC given a [90] laminate with a maximum applied load level of $40 \mathrm{MPa}$ and an R ratio of 0.1 . 


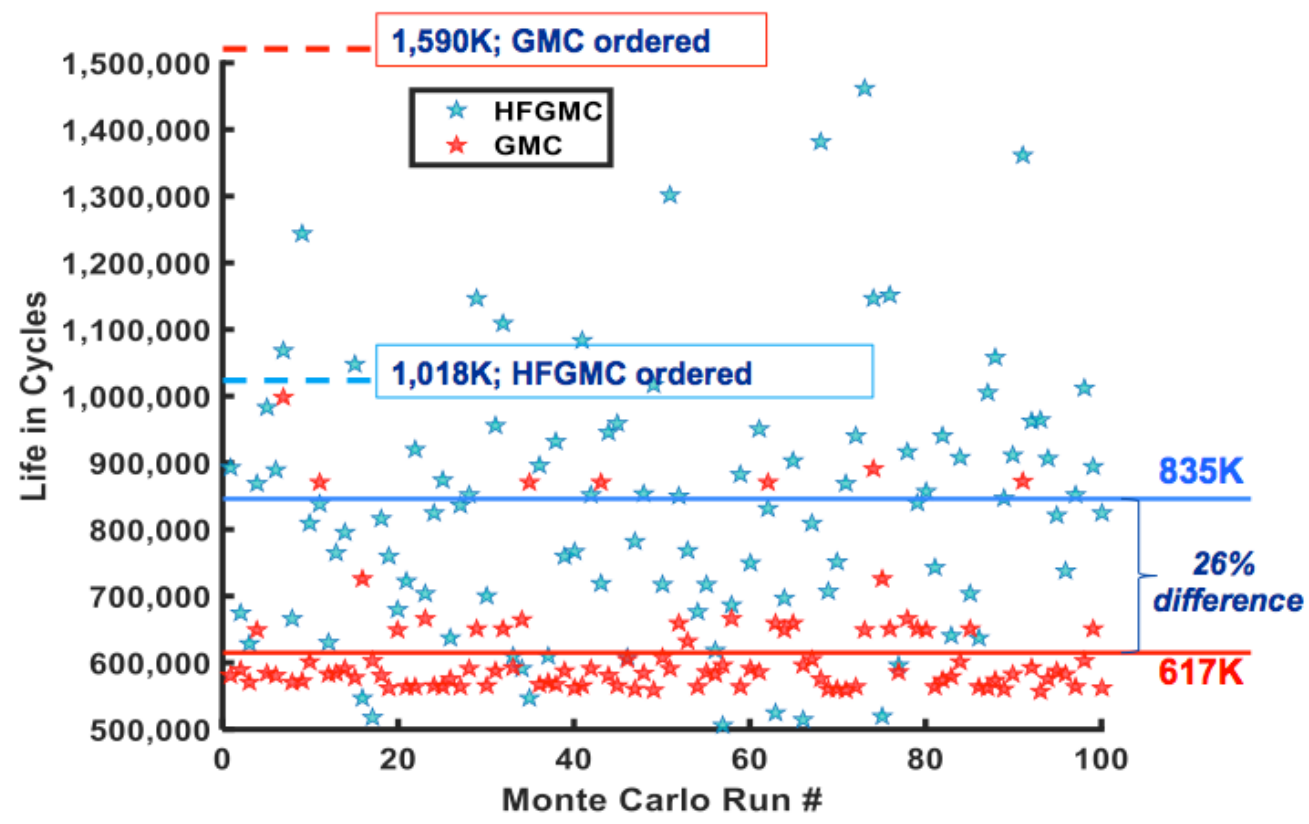

Figure 12 Final transverse fatigue lives as a function of run number for HFGMC (blue stars) and GMC (red stars) at $40 \mathrm{MPa}$ load level.

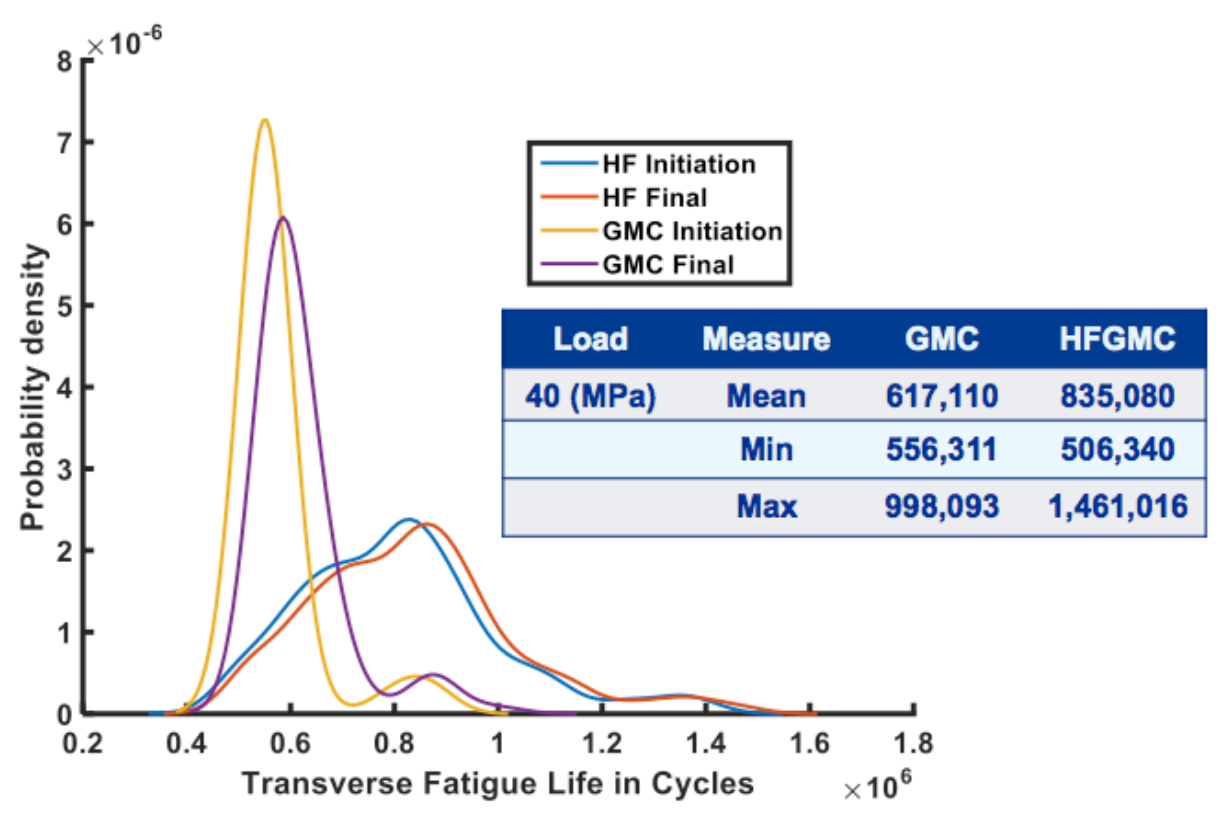

Figure 13: Probability density function representing initial and final fatigue failures, given a 4 fiber $24 \times 24$ RUC. 
Many observations can be made from these results. The variation in lives is much tighter for GMC than HFGMC, as the ratio of maximum to minimum lives is 1.8 for GMC and 2.88 for HFGMC. Further, the corresponding ordered microstructure life is $157 \%$ longer than the mean of the disordered microstructures in the case of GMC and only $22 \%$ different in the case of HFGMC. Interestingly, the difference between the mean life calculated using HFGMC (i.e., 835K cycles) and that using GMC (i.e., $617 \mathrm{~K}$ cycles) is only $26 \%$, whereas, for a given disordered realization, this difference is up to $230 \%$. This suggests that the far more efficient GMC (at least 1000 times faster than HFGMC) may still be useful to estimate averaged fatigue lives of disordered microstructures even though its local field accuracy and even effective properties are suspect in the case of a single disordered microstructure realization. This is particularly true since GMC is on the conservative side with respect to HFGMC in the case of disordered microstructures. This is opposite in the case of ordered microstructures, i.e., GMC predicts longer lives than HFGMC.

Similar trends hold for other load levels as well, see the S-N curves in Figures 14 and 15, where the results from the baseline ordered microstructure and results of the minimum and maximum life disordered microstructures at various applied load levels are shown. Figure 14 shows that, for GMC, the ordered case predicts a life outside the scatter range of the random disordered case, with a longer life than even the highest life case, within the 100 cases examined. Conversely, Figure 15 indicates that, in the case of HFGMC, the ordered microstructure falls within the scatter range of the various disordered microstructure cases. Also in Figure 15, X marks the life of our previously examined disordered Case 2 which has even a longer life than any of the 100 disordered cases randomly generated. This indicates that if more random microstructural cases were considered, the spread between maximum and minimum would increase even further than that shown in Figures 12 and 13 . The above results suggest that micromechanics can be used to inform the manufacturing process as to which microstructures can potentially provide maximum life for a given loading scenario. Whether or not such a microstructure could practically and reliably be obtained is still an open question.

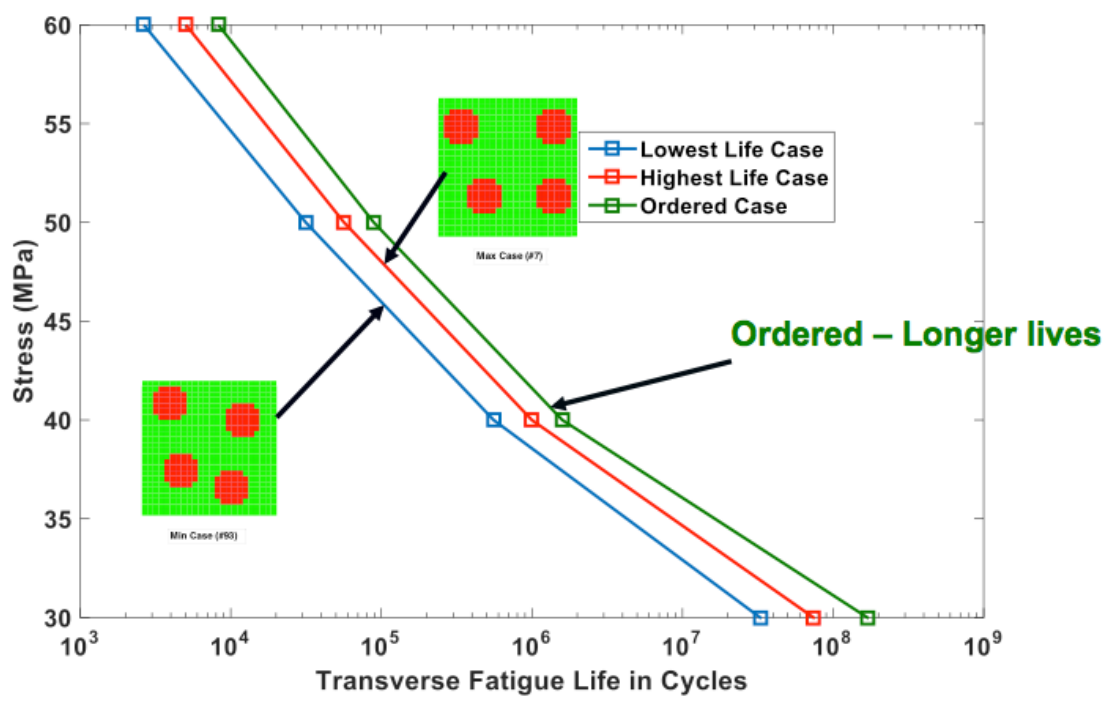

Figure 14: Ordered, minimum, and maximum disordered microstructure S-N Curves produced using GMC for a transversely loaded, unidirectional PMC laminate: $\mathrm{R}=0.1$ and $\mathrm{V}_{\mathrm{f}}=22 \%$. 


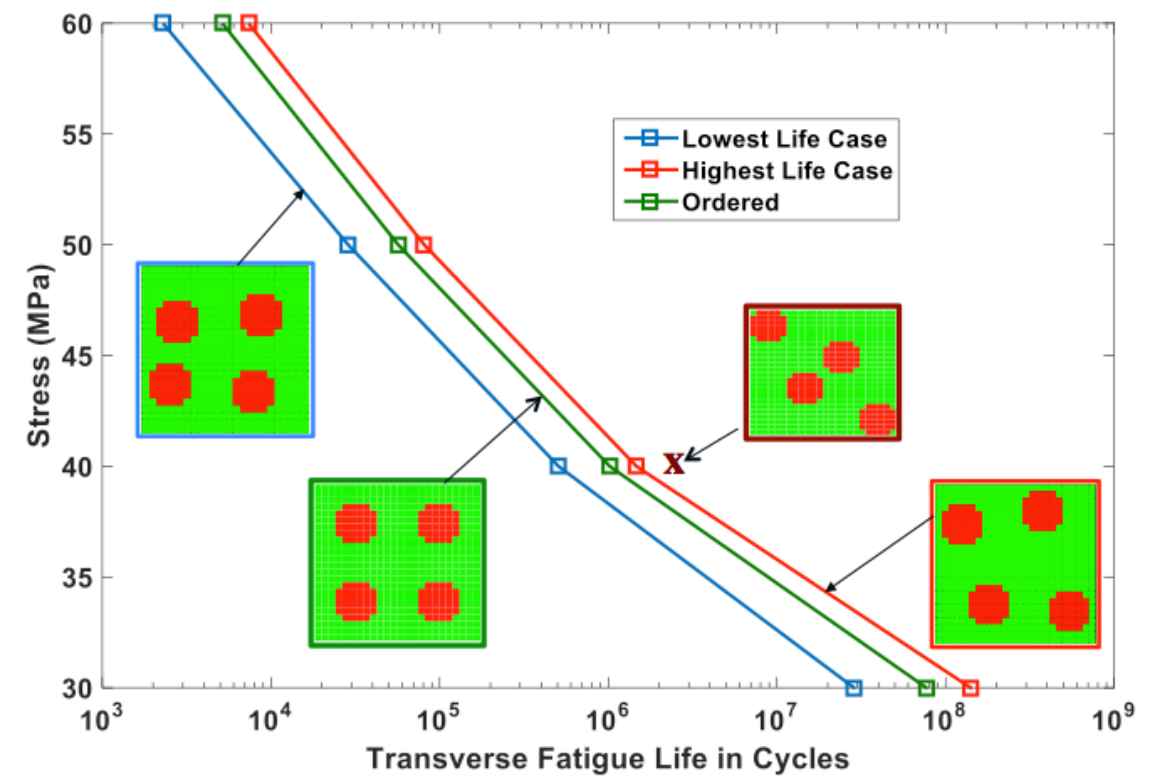

Figure 15: Ordered, minimum, and maximum disordered microstructure S-N Curves produced using HFGMC for a transversely loaded, unidirectional PMC laminate: $\mathrm{R}=0.1$ and $\mathrm{V}_{\mathrm{f}}=22 \%$.

\section{Conclusion}

ICME is an integrated approach to the design of products and the materials that comprise them by linking material models at multiple time and length scales. Manufacturing processes, which produce internal material structures, that in turn influence material properties, allowables, and responses can be tailored (engineered) to specific industrial applications. In this paper the statistical influence of microstructure (both ordered and disordered) on the unidirectional and laminated composite effective properties and fatigue life was investigated. In addition, the advantages/limitations of the micromechanics idealization (GMC or HFGMC) available within the general, synergistic, multiscalemodeling framework for composites (developed by the NASA Glenn Research Center (GRC) and known as MAC/GMC and FEAMAC) when considering microstructural arrangement was discussed. The important findings are summarized:

a) Accounting for spatial variations in composite microstructure within the RUC analyzed is important, as these variations can account for observed statistical variations in both effective properties and fatigue life.

b) Both micromechanics idealizations can be used to account for this variation; however, the more computationally efficient Generalized Method of Cells (GMC) is significantly less sensitive to microstructure variations than is the High Fidelity Generalized Method of Cells (HFGMC).

i. HFGMC typically predicted higher effective properties and lower fatigue lives than did GMC for order microstructures. Except for Arch $I D=1$, the difference in normal stiffness and Poisson's ratios were less than $0.5 \%$, while the differences in shear moduli were less than $10 \%$. Arch $I D=1$ should not be used with $\mathrm{HFGMC}$ as both out of plane shear moduli and fatigue lives can be significantly over estimated. 
ii. Predicted fatigue lives utilizing GMC with RUCs representing ordered microstructures are higher and significantly outside the range of those with disordered microstructures; whereas fatigue lives predicted with ordered RUCs using HFGMC are within the range of those lives determined using disordered microstructures.

iii. Although the fatigue lives predicted by HFGMC vary significantly based on individual microstructures (whereas GMC is relatively insensitive), the mean life value (i.e., averaged over multiple microstructure realizations) for a given RUC discretization (e.g., 4 (12x12) Fiber unit cells within the RUC) between HFGMC and GMC is relatively small $(<30 \%)$ compared to lives produced using an ordered (single fiber with periodic boundary conditions) microstructure. Note, that the actual percentage discrepancy, in the case of disordered microstructures, is highly dependent upon volume fraction and property mismatch between constituents.

Clearly, micromechanics can be effectively utilized to link the material microstructure (e.g., constituent phase properties, volume fraction, fiber packing (ordered or disordered), etc.) to ply/laminate properties (mesoscale) and finally to performance (at the macroscale), in an efficient and accurate manner to enable "fit-for-purpose" tailoring of the composite material. The ability to localize and homogenize efficiently between scales make MAC/GMC and FEAMAC ideal candidates for ICME simulations in a multiscale environment in which the microstructure can be optimized spatially based on the local loading and environmental history. Finally, extreme caution should be used when adopting/utilizing input constituent (fiber/matrix) properties either from the literature or a given model, as the fidelity of the given model used to obtain those "in-situ material properties" will impact the predictive ability of another model with a different degree of fidelity and assumptions.

\section{References:}

1. Trias, D., Costa, J., Turon, A., \& Hurtado, J. E. (2006). Determination of the critical size of a statistical representative volume element (SRVE) for carbon reinforced polymers. Acta Materialia, 54(13), 3471-3484.

2. Huang, Y., Jin, K. K., \& Ha, S. K. (2008). Effects of fiber arrangement on mechanical behavior of unidirectional composites. Journal of composite materials, 42(18), 1851-1871.

3. Maligno, A. R., Warrior, N. A., \& Long, A. C. (2009). Effects of inter-fibre spacing on damage evolution in unidirectional (UD) fibre-reinforced composites. European Journal of MechanicsA/Solids, 28(4), 768-776.

4. Wang, Z., Wang, X., Zhang, J., Liang, W., \& Zhou, L. (2011). Automatic generation of random distribution of fibers in long-fiber-reinforced composites and mesomechanical simulation. Materials \& Design, 32(2), 885-891

5. Romanov, V., Lomov, S. V., Swolfs, Y., Orlova, S., Gorbatikh, L., \& Verpoest, I. (2013). Statistical analysis of real and simulated fibre arrangements in unidirectional composites. Composites Science and Technology.

6. Garnich, M. R., Fertig, R. S., Anderson, E.M. (2013); "Random Fiber Micromechanics of Fatigue Damage", 54th AIAA/ASME/ASCE/AHS/SC Structures, Structural Dynamics, and Materials Conference, AIAA 2013-1656, Boston, MA, April 8-11, 2013.

7. Bednarcyk, B. A. and Arnold, S. M. , (2002); "MAC/GMC 4.0 User's Manual, Volume 2: Keywords Manual", TM 2002-212077/Vol 2, 2002

8. Aboudi, J., Arnold, S.M., Bednarcyk, B.A., (2013); Micromechanics of Composite Materials: A Generalized Multiscale Analysis Approach, Elsevier, Inc., 2013. 
9. Pineda, E.J., Bednarcyk, B.A., Arnold, S.M., (2014) “Achieving ICME with Multiscale Modeling: The Effects of Constituent Properties and Processing on the Performance of Laminated Polymer Matrix Composite Structures", AIAA SciTech, 2014, 55th AIAA/ASME/ASCE/AHS/SC Structures, Structural Dynamics, and Materials Conference.

10. Pineda, E.J., Bednarcyk, B.A., Waas, A.M. and Arnold, S.M.; (2013) "Progressive Failure of a Unidirectional Fiber-reinforced Composite Using the Method of Cells: Discretization Objective Computational Results", IJSS, Vol. 50, pp. 1203-1216

11. Paley, M. and Aboudi, J., (1992) "Micromechanical Analysis of Composites by the Generalized Cells Model, "Mechanics of Materials, Vol.14, 1992, pp.127-139.

12. Aboudi, J., Pindera, M.J. and Arnold, S.M. (2002) "Higher-Order Theory for Periodic Multiphase Materials With Inelastic Phases", NASA/TM 2002-211469. Also see IJP, 2003, Vol. 19, pp. 805-847.

13. Liu, K. C., Ghoshal, A. (2014). Inherent symmetry and microstructure ambiguity in micromechanics. Composite Structures, 108, February 2014, 311-318.

14. Arnold, S.M., Kruch, S., 1994. Differential Continuum Damage Mechanics Models for Creep and Fatigue of Unidirectional Metal Matrix Composites. International Journal of Damage Mechanics 3 (2), 170-91.

15. Chaboche, J.L. and Lesne, P.M., 1988. A Non-Linear Continuous Fatigue Damage Model, Fatigue Fract. Engng. Mater. Struct., Vol. 11, No. 1, pp. 1-7.

16. Plastics Design Library (1995) Fatigue and Tribological Properties of Plastics and Elastomers. Plastics Design Library, William Andrew, Inc., Norwich, NY.

17. Wilt, T.E., Arnold, S.M., Saleeb, A.F., 1997. A Coupled/Uncoupled Computational Scheme for Deformation and Fatigue Damage Analysis of Unidirectional Metal-Matrix Composites. Applications of Continuum Damage Mechanics to Fatigue and Fracture, ASTM STP 1315, D.L. McDowell (Ed.), 6582. 